\title{
ADENOSARCOMA DE ALTO GRADO DE ENDOCÉRVIX Y CAVIDAD ENDOMETRIAL CON COMPONENTE HETERÓLOGO DE RABDOMIOSARCOMA. REPORTE DE CASO Y REVISIÓN DE LA LITERATURA
}

\section{High grade adenosarcoma of endocervix and} ondometrial cavity with a heterologous component the literature

Zoraida Frías-Sánchez, $\mathrm{MD}^{1}$; Manuel Pantoja-Garrido, $\mathrm{MD}^{* 2}$; Álvaro Gutiérrez-Domingo, $\mathrm{MD}^{3}$; Julián Jiménez-Gallardo, $\mathrm{MD}^{2}$; Alfredo Polo-Velasco, MD, PhD'; Inmaculada Rodríguez-Jiménez, $\mathrm{MD}^{2}$

Recibido: septiembre 20/18 - Aceptado: junio 17/19

\section{RESUMEN}

Objetivo: presentar el caso de una paciente con diagnóstico de adenosarcoma de alto grado de endocérvix y de cavidad endometrial, con componente heterólogo, y hacer una revisión de la literatura con especial atención al diagnóstico y manejo terapéutico de esa patología.

Materiales y métodos: Se presenta el caso de una paciente de 31 años, que consulta al Hospital Universitario Virgen Macarena de Sevilla, institución regional de tercer nivel, por presentar sangrado genital originado por una masa polipoidea endocervical que se llevó a biopsia con resultado de un leiomiosarcoma poco diferenciado de alto

Correspondencia: Manuel Pantoja Garrido, Avenida Andalucía 53-55 1º. CP: 11007, Cádiz (España)Teléfono: 610035645 pantoja_manuel@hotmail.com

1 Unidad de Gestión Clínica de Obstetricia y Ginecología del Hospital Universitario Virgen del Rocío, Sevilla (España).

2 Unidad de Gestión Clínica de Obstetricia y Ginecología del Hospital Universitario Virgen Macarena, Sevilla (España).

3 Unidad de Gestión Clínica de Anatomía Patológica del Hospital Virgen Macarena, Sevilla (España). grado de endocérvix. Posteriormente fue llevada a histerectomía total abdominal. En el estudio de la pieza quirúrgica el resultado fue: adenosarcoma de endocérvix y cavidad endometrial, con componente heterólogo de rabdomiosarcoma. Con los términos: “adenosarcoma”, “endocervical”, “cérvix”, “uterus”, "heterologous", en las bases de datos Medline vía PubMed se realizó una búsqueda de artículos de revisión bibliográfica, reportes y series de casos clínicos que describían aspectos del adenosarcoma cervicouterino y del componente heterólogo de rabdomiosarcoma, en inglés y español, publicados desde 1974.

Resultados: se hallaron seis artículos correspondientes a revisiones de la literatura, reportes o series de casos, donde se describen los aspectos más importantes referentes al diagnóstico y tratamiento de esta patología.

Conclusiones: esta patología se caracteriza, en ocasiones, por un crecimiento rápido y agresivo, donde es importante el diagnóstico precoz y el 
tratamiento óptimo, basado en una combinación de cirugía, radioterapia y quimioterapia, aunque dada su baja prevalencia se necesitan aún más estudios para poder confirmar estos datos.

Palabras clave: adenosarcoma; endocervical; cérvix; útero; heterologous.

\section{ABSTRACT}

Objective: To present the case of a patient diagnosed with high grade adenosarcoma of the endocervix and the endometrial cavity, with a heterologous component, and to conduct a review of the literature focusing on the diagnosis and therapeutic management of this disease condition.

Materials and methods: We present the case of a 31-year-old female patient who came to Virgen Macarena University Hospital of Seville, a Level III regional institution, complaining of genital bleeding arising from an endocervical polypoid mass. The biopsy of the mass revealed a high grade, poorly differentiated leiomyosarcoma of the endocervix. The patient was taken later to total abdominal hysterectomy. The study of the surgical specimen provided the following result: adenosarcoma of the endocervix and endometrial cavity with a heterologous rhabdomyosarcoma component. A search was conducted in the Medline database via Pubmed using the terms "adenosarcoma," "endocervical," "cervix," "uterus," "heterologous." The search included literature review articles, case reports and clinical case series describing aspects of cervical adenosarcoma and the heterologous rhabdomyosarcoma component, published in English and Spanish since 1974.

Results: Six articles corresponding to literature reviews, case reports or case series in which the most relevant aspects of the diagnosis and treatment of this disease condition are described were retrieved.

Conclusions: This condition is characterized, on occasions, by rapid and aggresive growth, hence the importance of early diagnosis and optimal treatment based on a combination of surgery, radiation therapy and chemotherapy. However, due to its low prevalence, further studies are needed in order to confirm these data.

Key words: Adenosarcoma; endocervical; cervix; uterus; heterologous.

\section{INTRODUCCIÓN}

El adenosarcoma (AS) es un tumor raro compuesto por epitelio glandular benigno y componente mesenquimal maligno (1). Fue descrito en el año 1974 por Clement y Scully, y se definió como adenosarcoma mülleriano (AM) (2). Se estima una prevalencia de entre el 5-8\% de todos los sarcomas uterinos, aunque puede también originarse con menor frecuencia en ovario, vagina, trompas o el peritoneo intestinal. Su localización en el cérvix y la presencia de elementos heterólogos se considera extremadamente rara. Las manifestaciones clínicas y las pruebas radiológicas no son específicas. El síntoma más común es el sangrado vaginal irregular asociado, en muchos casos, a la existencia de una masa polipoidea única que protruye a través del cérvix uterino, la cual se confirma con las pruebas de imagen, que pueden identificar los límites de la formación y la invasión de tejidos adyacentes (3, 4). Sin embargo, el diagnóstico definitivo se basa en el estudio histopatológico de la pieza quirúrgica $(1,5)$, ya que puede pasar inadvertido en la biopsia, confundiéndose con pólipos o miomas submucosos en el estudio prequirúrgico. En cuanto al manejo, no hay claridad sobre el papel del tratamiento quirúrgico, la quimio o la radioterapia coadyuvante (1).

Dada la baja frecuencia de este tumor es importante que el ginecólogo, el oncólogo y el patólogo conozcan los aspectos más importantes de su manejo. Por tanto, se presenta el caso de una paciente diagnosticada con adenosarcoma de endocérvix y cavidad endometrial, con componente heterólogo de rabdomiosarcoma, con el objetivo de realizar una revisión de la literatura respecto al diagnóstico clínico, radiológico e histológico, y sobre las alternativas terapéuticas utilizadas para su manejo en la actualidad; además, se aportan algunos datos 
sobre los aspectos generales o el pronóstico de esta infrecuente entidad clínica.

\section{REPORTE DE CASO}

Paciente de 31 años, remitida de una consulta externa de carácter privado al Servicio de Urgencias Ginecológicas del Hospital Universitario Virgen Macarena, institución hospitalaria regional pública de tercer nivel en la provincia de Sevilla, España, por presentar numerosos episodios de metrorragias intermenstruales de cuantía abundante. Como antecedente familiar de interés oncológico refería que una tía materna había padecido cáncer de mama detectado después de los 50 años, y tenía historia de única gestación previa que finalizó en parto eutócico sin complicaciones, sin otros antecedentes de importancia. A su llegada a Urgencias se encontraba en buen estado general y estable hemodinámicamente; se confirma la existencia de una metrorragia abundante que procedía de una formación polipoidea y exofítica que protruía a través del cérvix. Ante dicha situación, se tomó biopsia de la formación, se coaguló la zona sangrante y se colocó un taponamiento vaginal que, finalmente, contrarrestó el sangrado, por lo que la paciente fue dada de alta al día siguiente. Fue citada a los 10 días a consulta de Ginecología Oncológica para control, donde se le realizó una exploración completa. Se observó un abdomen blando y depresible, sin apreciarse masas ni megalias, y genitales externos normales; en la especuloscopia se evidenció un ectocérvix de aspecto normal, aunque el orificio cervical externo se encontraba dilatado por una formación exofítica de aspecto polipoideo-neoplásico. En el tacto combinado, el cérvix estaba globalmente engrosado por la ocupación endocervical, con cuerpo uterino normal. La exploración se complementó con una ecografía transvaginal, en la que se confirmó un endocérvix ocupado por una formación de unos 2,5 cm, que no parecía afectar al istmo. La línea endometrial mostraba en ese momento un aspecto normal. El resultado de la biopsia obtenida en urgencias fue "leiomiosarcoma poco diferenciado (de alto grado)". En dicha consulta se solicitó una resonancia magnética (RM) abdominopélvica, donde se confirmó la presencia de una lesión endocervical neoplásica descrita como: "la cavidad de endocérvix está distendida por una lesión de tejido sólido heterogéneo que mide 25-30 mm y es compatible con neoplasia. No se observa invasión del estroma cervical ni extensión al útero, vagina o anexos. No se observan ascitis ni adenopatías" (figura 1). Así pues, se clasificó como sarcoma de endocérvix estadio IB1 según la clasificación de la Federación Internacional de Ginecología y Obstetricia (FIGO) de 2016. Posteriormente, se discutió el caso en el Comité de Tumores Ginecológicos, donde se planteó por unanimidad evitar la ooforectomía bilateral dada la edad de la paciente, ya que el pronóstico no se presuponía diferente en ese caso. Así pues, se le realizó una laparotomía media infraumbilical con histerectomía total y salpinguectomía bilateral, sin complicaciones intraoperatorias. Durante su estancia hospitalaria, la evolución fue excelente, con una reincorporación precoz y progresiva a sus actividades cotidianas. Recibió el alta domiciliaria a los 4 días de la intervención. A los 15 días se citó en la consulta para revisión posquirúrgica y para informar el resultado de patología quirúrgica: "Adenosarcoma de endocérvix y cavidad endometrial, con componente heterólogo de rabdomiosarcoma". El comentario anatomopatológico añadido al informe lo detalla de forma específica: "La neoplasia tiene predominantemente un crecimiento polipoideo exofítico, constituida exclusivamente por un crecimiento fusocelular sarcomatoide de alto grado, que expresa desmina, actina muscular y miogenina. En este crecimiento prácticamente no se identifican estructuras glanduliformes. El diagnóstico es el de sarcoma muscular con diferenciación estriada: rabdomiosarcoma" (figura 2). Así pues, fue derivada a la Consulta de Oncología Médica, donde se propuso una pauta de quimioterapia con DocetaxelGemcitabina y radioterapia adyuvante en la pelvis y ganglios pélvicos bilaterales. La paciente realizó los dos primeros ciclos de quimioterapia, tras los 


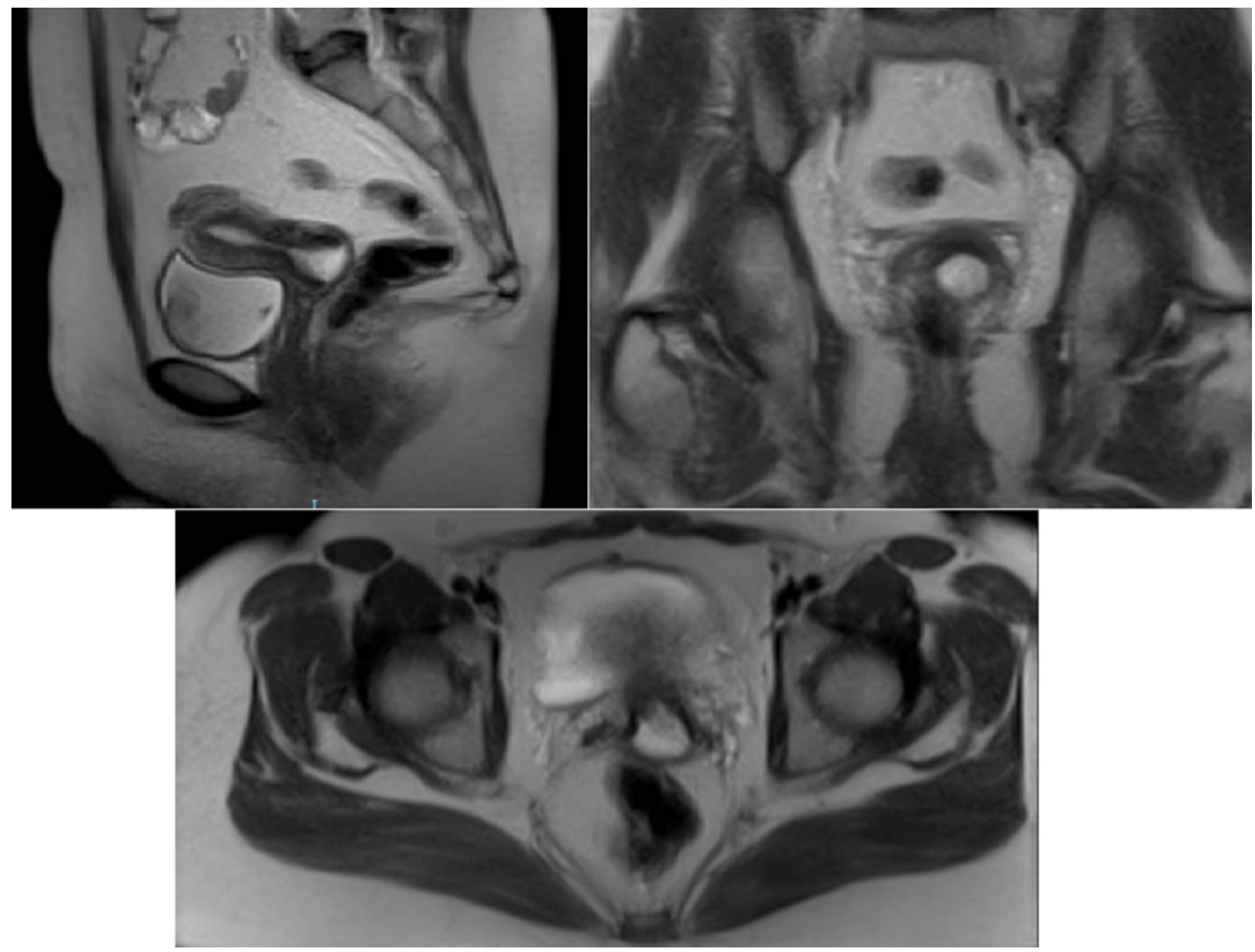

Figura 1. Imágenes de RM, donde se observa una masa de 25-30 mm, que distiende el canal endocervical en una mujer con un adenosarcoma de endocérvix y endometrio

cuales rechazó continuar con tratamientos coadyuvantes (incluida la radioterapia). El seguimiento se realizó de forma parcial e incompleta, ya que solo deseaba ser valorada por el equipo de Ginecología Oncológica, rechazando continuar con cualquier otro seguimiento, tanto en Oncología Radioterápica como en Oncología Médica. Los controles posteriores realizados desde la intervención (hace 4 años), y las pruebas complementarias solicitadas han sido normales; actualmente se encuentra libre de enfermedad (última consulta, 2019).

Aspectos éticos. Se solicitó autorización tanto a la paciente como al Comité de Ética en Investigación del Hospital Universitario Virgen Macarena para la publicación de las imágenes y la información referente al proceso clínico. Se garantizó la confidencialidad tanto de la información como de la identificación de la paciente, para proteger sus derechos de privacidad.

\section{MATERIALES Y MÉTODOS}

Se realizó una búsqueda bibliográfica en las bases de datos Medline vía PubMed, utilizando los términos: “adenosarcoma”, “endocervical”, “cérvix”, “uterus”, "heterologous"; se buscaron artículos de revisión bibliográfica y estudios de reporte de casos y series de casos que describian aspectos del adenosarcoma cervicouterino, su posible presentación polipoidea y del componente heterólogo de rabdomiosarcoma, en inglés y español, publicados desde 1974.

\section{RESULTADOS}

Se identificaron 38 artículos, de los cuales se incluyeron 6 publicaciones que cumplieron con los crite- 

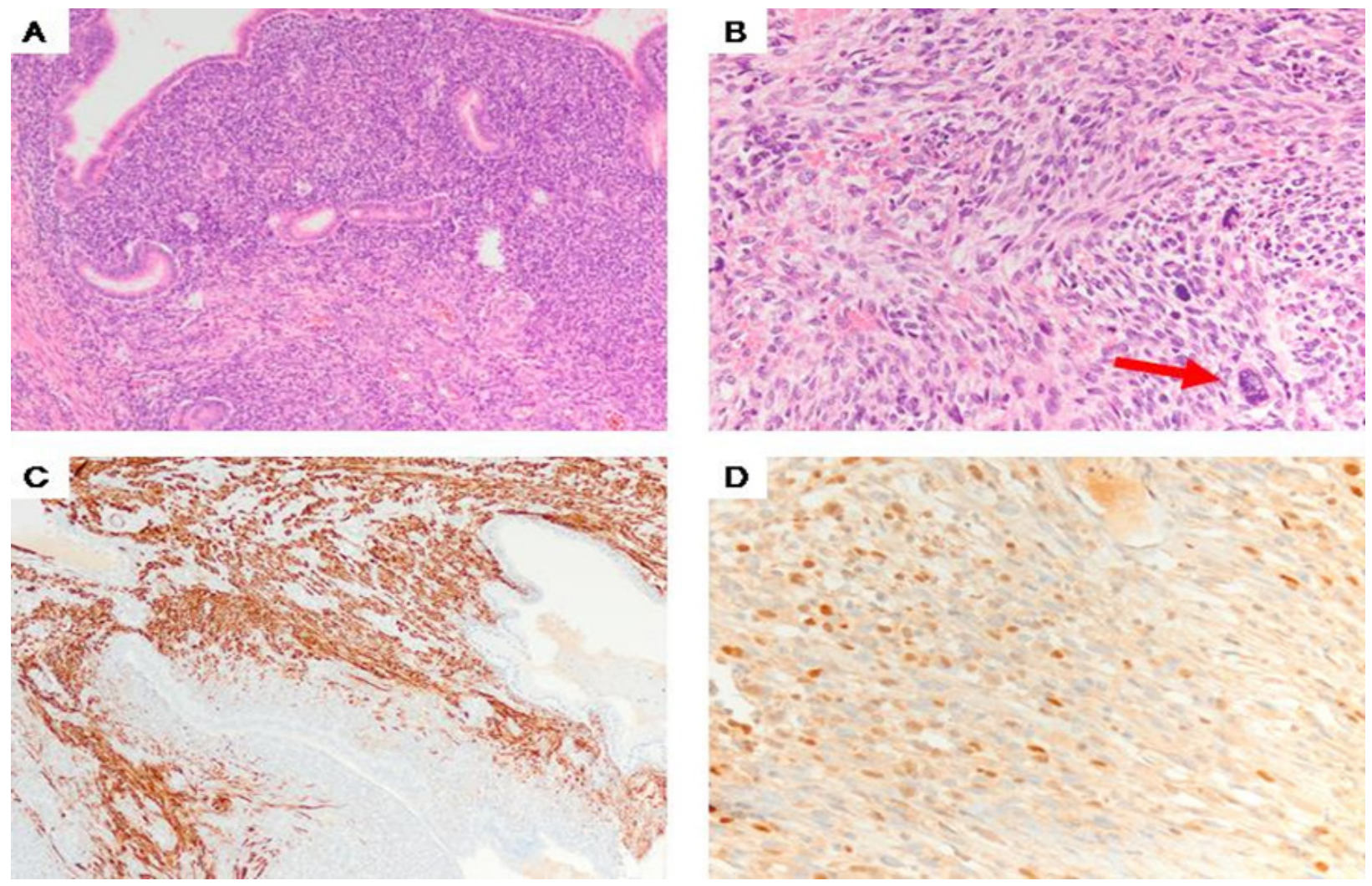

Figura 2. Adenosarcoma con componente heterólogo de rabdomiosarcoma. A) A poco aumento se aprecia un crecimiento fusocelular atípico en torno a estructuras glandulares (HE, 4x). B) A mayor aumento, y en un área adyacente a la anterior, se aprecia un área sarcomatoide caracterizada por células de hábito fusiforme, alguna de ellas con marcada atipia (flecha) y frecuentes figuras de mitosis (HE, 20x). C) Inmunorreacción positiva para desmina (marcador muscular) en el componente mesenquimal, negativo en el componente epitelial (glandular) (desmina, 10x). D) Inmunorreacción positiva focal para miogenina en el componente mesenquimal. Esto nos pone de manifiesto la presencia de componente heterólogo de rabdomiosarcoma (miogenina, 20x)

rios de búsqueda (1-6). Los artículos seleccionados corresponden a reportes de casos o series de casos clínicos (2, 4), revisiones de la literatura $(3,5,6)$, y revisiones de la literatura a partir de casos clínicos (1). Todos los artículos son publicaciones en inglés.

Diagnóstico clínico. Las manifestaciones clínicas no son específicas, el síntoma más común es el sangrado vaginal irregular asociado, en muchos casos, a la existencia de una masa polipoidea que protruye a través del cérvix uterino (6).

Diagnóstico histológico. Las características histológicas que permiten diferenciar un adenosarcoma de adenofibromas o de pólipos endocervicales han sido descritas por Clement y Scully (2), y Zaloudek y Norris (4), y son las siguientes: 1) un marcado grado de atipia de las células mesenquimales, 2) elementos heterólogos con carácter maligno, 3) presencia de invasión miometrial, 4) cuatro o más figuras mitóticas por cada diez HPF (high-power fields) $(2,4)$. La tinción más frecuentemente utilizada para los estudios histológicos e inmunohistoquímicos fue la hematoxilina-eosina (1). El epitelio glandular y la proliferación mesenquimal con atipias características se observó en todos los trabajos revisados (1-6), asociado a componentes heterólogos $(1,4)$, en algunas revisiones hasta en un $20 \%$ de pacientes (6), como el de rabdomiosarcoma, con inmunorreación a desmina-actina e invasión vascular (1). Los focos necróticos o hemorrágicos, o las formas polipoideas/ submucosas de las neoplasias se observaron en un 
solo artículo (1). En un estudio se observaron $\geq 4$ figuras mitóticas por cada 10 HPF (4), y en otro (1), valores inferiores a dicha cifra. Desde el punto de vista inmunohistoquímico no hubo reacción de las células estromales/mesenquimales a CD10 en ningún trabajo, ni otras expresiones como Ki67, p53, p16 o MNF116.

Diagnóstico mediante técnicas de imagen. la ultrasonografía pélvica fue realizada en todos los trabajos revisados (1-6).

Estrategias terapéuticas. La terapia óptima para los adenosarcomas es aún desconocida (1). En principio, se debe plantear la intervención en función de la edad y los factores de riesgo de la paciente; lo recomendado por la mayoría de los autores es la histerectomía total con anexectomía bilateral (4). No obstante, aún es poco claro si la anexectomía debe formar parte sistemáticamente del tratamiento, así pues, en mujeres jóvenes puede plantearse una cirugía con conservación de los ovarios (3). El estudio linfático es un punto controvertido en este tema, ya que no se sabe con certeza la utilidad que pueda tener y su repercusión en el tratamiento adyuvante. Algunos autores sugieren que la linfadenectomía puede no ser necesaria en pacientes con tumor confinado al útero y sin factores de riesgo (5). Hay ocasiones en las que el estudio linfático puede determinar el pronóstico y la necesidad de terapia adyuvante (3), así pues, en aquellos pacientes con crecimiento sarcomatoso, invasión miometrial profunda, invasión linfovascular y estadio alto, podría considerarse realizar la linfadenectomía (6). El tratamiento adyuvante en este tipo de patologías no está bien establecido, por lo que se mantiene el régimen estándar utilizado en los sarcomas: doxorrubicina, ifosfamida o gemcitabina/docetaxel (5) (como ocurre en nuestro caso). En nuestra revisión, todas las pacientes se sometieron a cirugía (1-6), en una de ellas se conservó un ovario (2), además de un caso de histerectomía simple por vía vaginal (2). La vía de elección mayoritaria fue la laparotomía. La linfadenectomía asociada, tanto pélvica como aortocava, es infrecuente, aunque en una revisión de 994 pacientes se encontró hasta un 2,9\% de metástasis linfáticas en pacientes con adenosarcoma (3). Los diferentes trabajos describen distintas terapias adyuvantes. Cuatro estudios aportan datos y experiencias sobre el tratamiento con quimioterapia $(1,3,4,6)$, con revisiones en las que se indican porcentajes de tratamiento de un 11,3 a un 30\% de las pacientes $(3,6)$, única o combinada con radioterapia. La radioterapia también es una forma de tratamiento frecuente hasta en cuatro de los trabajos revisados $(2,3,4,6)$, en algunos casos combinada con quimioterapia $(3,6)$ o braquiterapia (6).

Pronóstico. Los principales factores de riesgo que afectan directamente la tasa de recidiva y el potencial metastásico son los ya mencionados (6), junto al tamaño del tumor, la enfermedad extrauterina, el tipo histológico y, fundamentalmente, el crecimiento sarcomatoso del tumor (3). Se estima una tasa de recidiva del 15-25\% cuando hablamos de un AS sin crecimiento sarcomatoso, y un 45-70\% cuando se trata de un AS con crecimiento sarcomatoso (6).

\section{CONCLUSIONES}

El AS se considera un tumor de aparición muy infrecuente; más raro aún es el AS de alto grado con componentes heterólogos. En muchos casos posee un crecimiento rápido y agresivo, por lo que el diagnóstico precoz y el tratamiento óptimo son fundamentales. El diagnóstico se puede ver dificultado por la similitud con patologías benignas como los pólipos endometriales o miomas, con los que se confunde en algunos casos. El tratamiento adecuado es aún desconocido por su baja prevalencia, sin embargo, se postula una combinación de cirugía, radioterapia y quimioterapia, tratándolo como un adenosarcoma de alto grado por su composición histológica heteróloga.

\section{REFERENCIAS}

1. Ramos P, Ruiz A, Carabias E, Piñero I, Garzón A, Álvarez I. Müllerian adenosarcoma of the cervix with heterologous elements: Report of a case and review of the literature. Gynecol Oncol. 2002;84(1):161-6. doi: https://doi.org/10.1006/gyno.2001.6423. 
2. Clement PB, Scully RE. Mullerian adenosarcoma of the uterus. A clinicopathologic analysis of ten cases of a distinctive type of mullerian mixed tumor. Cancer. 1974;34(4):1138-49.

3. Machida H, Nathenson MJ, Takiuchi T, Adams CL, Garcia-Sayre J, Matsuo K. Significance of lymph node metastasis on survival of women with uterine adenosarcoma. Gynecol Oncol. 2017;144(3):524-30. doi: 10.1016/j.ygyno.2017.01.012.

4. Zaloudek CJ, Norris HJ. Adenofibroma and adenosarcoma of the uterus: A clinicopathologic study of 35 cases. Cancer. 1981;48:354-66.
5. Tanner EJ, Toussaint M, Leitao MM, Hensley ML, Soslow RA, Gardner GJ, et al. Management of uterine adenosarcomas with and without sarcomatous overgrowth. Gynecol Oncol. 2013;129(1):140-4. doi: 10.1016/j.ygyno.2012.12.036.

6. Caroll A, Ramirez PT, Westin SN, Soliman PT, Munsell MF, Nick AM, et al. Uterine adenosarcoma: An analysis on management, outcomes, and risk factors for recurrence. Gynecol Oncol. 2014;135:455-61. doi: 10.1016/j.ygyno.2014.10.022.

\section{Conflicto de intereses: ninguno declarado.}

